\title{
Resultados obtenidos a largo plazo de un programa de prevención de violencia de género en El Salvador
}

Laura Navarro-Mantas

Recibido: 01/11/2013 - Aceptado: 14/12/2013

\begin{abstract}
Resumen
El presente trabajo describe un programa de formación para futuros profesionales de la Psicología basado en investigaciones científicas sobre sexismo y poder. El programa fue desarrollado en una universidad salvadoreña y sus objetivos fueron: a) incrementar la conciencia sobre las desigualdades de género en la vida diaria; b) reducir las creencias sexistas ambivalentes; c) proporcionar a los participantes recursos y materiales útiles para reducir el sexismo, la violencia sexual y las actitudes homófobas en intervenciones sociales. El curso de formación duró 21 horas. Evidencias empíricas iniciales apoyan la efectividad del programa para la reducción de actitudes sexistas y el reconocimiento de la violencia de tanto inmediatamente después de la intervención como a los 6 meses.
\end{abstract}

\section{Palabras clave}

Violencia de género, sexismo, prevención, formación.

\begin{abstract}
The present work describes a gender training program for future professionals in Psychology based on scientific research on sexism and power. The training course was held in a Salvadorean University and aimed at: a) increasing awareness of gender inequalities in everyday life; b) reducing ambivalent sexist beliefs; c) providing participants with useful resources and materials to reduce sexism, sexual violence and homophobic attitudes in social interventions. The training course lasted 21 hours. Empirical evidence supports the effectiveness of the program to reduce participants' sexism and increase gender violence awareness both immediately after the intervention and 6 months later.
\end{abstract}

\section{Keywords}

Gender violence, sexism, prevention, training

\section{Introducción}

En la "Declaración sobre la eliminación de la violencia contra la mujer" de las Naciones Unidas (Res. A.G. 48/104, ONU, 1994), se definía la violencia de género como "todo acto de violencia basado en el género que tiene como resultado posible o real un daño físico, sexual o psicológico, incluidas las amenazas, la coerción o la privación arbitraria de la libertad, ya sea que ocurra en la vida pública o en la vida privada". Esta definición aclararía mucha de la controversia que existe, tanto en el ámbito académico, legislativo, medios de comunicación como en otros foros de discusión o espacios de la vida cotidiana en El Salvador sobre el significado de la terminología "violencia de género" y el análisis de este grave problema social. Se discute si esta nomenclatura hace referencia tanto al maltrato hacia los hombres como hacia las mujeres o, si por el contrario, se refiere exclusivamente a la violencia ejercida contra las mujeres. Dicha discusión surge por considerarse que género es una categoría que puede ser masculino y femenino. Sin 
embargo, la declaración de la ONU (1994) establece que violencia de género es "todo acto de violencia basado en el género", es decir, podemos entender que se refiere a la violencia que tiene una causa ideológica basada en la desigualdad histórica que han sufrido las mujeres en nuestras sociedades, y, por tanto, "violencia de género" se referiría a la violencia que sufren las mujeres por el simple hecho de ser mujeres. Este principio se vería refrendado por la misma Declaración de las Naciones Unidas, que añade que la violencia basada en el género se debe a la condición social, económica y jurídica de subordinación de las mujeres, convirtiendo esta condición en un problema grave de salud pública, violación de los derechos humanos y una barrera al desarrollo económico de los países.

Por añadidura, el "poder" adquiere un papel central en la declaración de Beijing (Naciones Unidas, 1995), como un componente central en la explicación de las causas de la violencia contra las mujeres, identificando esta violencia como "una manifestación de las relaciones de poder históricamente desiguales entre hombres y mujeres". Es por ello que el concepto de ha ido cobrando relativa importancia en las últimas décadas como variable socioestructural en distintas investigaciones psicológicas y de las ciencias del comportamiento; y resulta lógico tener como referencia su análisis y estudio en cualquier estrategia de prevención e intervención de la violencia de género.

Para llevar a cabo cualquier estrategia de prevención e intervención, así como la articulación de políticas públicas, es crucial contar con una estimación más o menos precisa de la magnitud de este problema social que es la violencia contra las mujeres, por lo que siempre debemos iniciar tomando en consideración las estadísticas nacionales e internacionales y otros registros que nos muestren, con pretendida rigurosidad, la prevalencia de la violencia que sufren las mujeres así como los tipos de maltrato que experimentan. Obtener este dato no es tarea fácil, teniendo en cuenta que es un fenómeno que ocurre en el ámbito privado y que a menudo hay creencias y patrones culturales que impiden u obstaculizan que salgan a la luz. En este sentido, cabe destacar los datos obtenidos en la Encuesta Nacional de Salud Familiar (Fesal, 2008) en la que el $91 \%$ de las mujeres entrevistadas reconocía que los problemas de pareja deben ser conversados solo dentro del hogar. Esta creencia tan arraigada aumenta la condición de vulnerabilidad de las mujeres, que en muchas de las ocasiones sufren en silencio el maltrato sin que puedan recibir ayuda de ningún familiar ni instancia pública o privada. Por otra parte, en las ocasiones en que otros familiares o vecinos son conocedores de que una mujer está sufriendo violencia en El Salvador, suelen considerar que no deben intervenir por tratarse de un tema privado.

Algunos datos mundiales de relevancia, en relación con los índices de maltrato que sufren las mujeres, son los del Estudio Multipaís de la OMS (2006), que en su informe publicaba los resultados obtenidos de diversos estudios llevados a cabo en diez países. En este informe se establecía que entre 15 y $71 \%$ de las mujeres de 15 a 49 años, de los diferentes países que llevaron a cabo el estudio, refirieron haber sufrido violencia física y/o sexual por parte de su pareja en algún momento de su vida (Estudio Multipaís de la OMS, 2006). En otro informe publicado recientemente por la Organización Panamericana de la Salud (2013), se recogen los datos de doce países latinoamericanos entre Ios que se encuentran Nicaragua, Guatemala y El Salvador. Según este informe, entre el 17 y el $53 \%$ de las mujeres latinoamericanas ha sufrido violencia física o sexual por su pareja alguna vez en la vida. Si dirigimos nuestra mirada a la realidad concreta de El Salvador, este país encabezó las cifras mundiales de feminicidios con un total de 647 asesinatos de mujeres en el año 2011; más de un tercio se registró en la capital; y el $49 \%$ de estos fue a mujeres de edades comprendidas entre los 18 y los 39 años (Ormusa, 2012). Dichas cifras muestran tristemente la gravedad de la situación de las mujeres en El Salvador, pero también dejan ver que es un problema que sufren a menudo las mujeres jóvenes. A su vez, el 56 \% de las mujeres salvadoreñas que ha tenido pareja en El Salvador ha sufrido algún tipo de maltrato o comportamientos controladores por parte de esta, siendo un $24 \%$ las mujeres que informaron de haber experimentado violencia física (Fesal, 2008). Y el $12 \%$ informó haber sufrido violencia sexual por parte de su pareja actual o anterior.

En esta misma encuesta se obtienen otros datos significativos relativos a las creencias y los valores que pueden sostener estos índices de violencia, pero que deben ser considerados con precaución porque se puede caer en el error de pensar que son creencias que tienen las mujeres y que, por ello, ellas son responsables del maltrato que sufren, como a menudo ocurre en el imaginario colectivo salvadoreño, ya que la investigación se hizo solo con mujeres. No obstante, de esta forma estaríamos culpabilizando y responsabilizando a las mujeres víctimas de la violencia que sufren y no estaríamos reconociendo que son creencias compartidas por hombres y mujeres y que, por tanto, es la sociedad en su conjunto la que tiene dicha responsabilidad. Así, llama la atención que el $43 \%$ de las mujeres opinara que una buena esposa debe obedecer a su esposo. Sería interesante, en futuros estudios, indagar sobre las características que un hombre considera debe tener toda mujer. A su vez, se encontró en esta 
investigación que el acuerdo con actitudes tradicionales, como que una mujer no tiene derecho a esterilizarse si el esposo no está de acuerdo y que si es maltratada por su esposo no debe intervenir nadie externo a la familia, fue mayor en las zonas rurales y en los departamentos (en torno al $50 \%$ ) que en la capital. Solo un $4 \%$ de las mujeres entrevistadas en esta encuesta no manifestó ninguna de estas creencias tradicionales.

Algunos autores consideran que parte de estas creencias compartidas en el imaginario colectivo de la sociedad salvadoreña se deben a una idiosincrasia basada en unos rígidos estereotipos de género en los que el papel masculino supone una referencia patriarcal que respalda aún el derecho del marido de "corregir" a su pareja. Por tanto, es una autoridad social (Buvinic, Morrison, y Shifter, 1999; Hume, 2008). Por otra parte, el papel femenino se considera de "naturaleza" y se fundamenta en unos pilares básicos como la maternidad, la afectividad, el cuidado de los demás y una sexualidad heterosexual y monógama. El papel femenino sigue además conservando como valor social la virginidad (Garaizabal y Vázquez, 1994).

Sin embargo, a pesar de la resistencia ideológica y cultural de la sociedad de El Salvador, en relación con el marco legal que aborda esta problemática en el país, en la actualidad cabe señalar que se han producido algunos cambios importantes que avanzan en la búsqueda de una solución al problema de la violencia contra las mujeres en El Salvador. Este avance tiene que ver con la aprobación de la Ley especial integral para una vida libre de violencia para las mujeres (LEIV), el 25 de noviembre de 2010 (Asamblea Legislativa, República de El Salvador, 2011), puesta en vigor en enero del año 2012. Esta ley es un hito, ya que como novedad brinda un nuevo abordaje de la violencia basada en el género frente a la Ley contra la Violencia Intrafamiliar que estaba vigente desde 1996 (Asamblea Legislativa, República de El Salvador, 2011), y que incluye otros tipos de violencia que ocurren en el hogar dirigidos a otros miembros de la unidad familiar, por lo que no contempla las circunstancias concretas en las que se produce la violencia de género y queda deficiente. La LEIV reconoce tres ejes importantes: la persecución y la sanción, la atención especializada y la prevención (Red Feminista Frente a la Violencia Contra las Mujeres, RED FEM, 2012). En esta ley se describen, como una forma de prevención, las normas y políticas formuladas para reducir la violencia contra las mujeres, interviniendo desde las causas identificadas de esta. Por tanto, dicha ley insta a la política nacional a promover programas que fomenten el "desaprendizaje" de los modelos convencionales que históricamente han sido atribuidos a las mujeres, y, concretamente, dentro de las responsabilidades ministeriales, el Ministerio de Educación debe establecer estos procesos de enseñanza y aprendizaje formales y no formales en todos los niveles educativos. A su vez, la política nacional, según se indica en la ley, para su cumplimiento e implantación deberá contener programas de sensibilización, conocimiento y especialización, para el personal que preste sus servicios en esta área, por lo que urge el diseño e implantación de programas de formación en violencia de género que se desarrollen tanto en estos ámbitos de trabajo como en el ámbito universitario por su responsabilidad en la formación de futuros profesionales.

Por otra parte, la política nacional de las mujeres de El Salvador y las demandas nacionales y compromisos internacionales en materia de género (Isdemu, 2011) plantean la necesidad de brindar una educación no sexista que, junto con otro tipo de políticas, contribuyan de manera efectiva a la construcción de una nueva ideología basada en la igualdad y la equidad, educación que debe erradicar el papel de subordinación de las mujeres, privilegiando la igualdad de condiciones para su desarrollo y su inclusión. A su vez, la Conferencia Regional de Educación Superior (Crees, 2008) ha hecho un llamado urgente y enfático a los miembros de las comunidades educativas de educación superior, sobre todo a los encargados de tomar decisiones políticas y estratégicas a favor de una formación que incida en el desarrollo de la región, haciendo notar, entre los aspectos, trabajar la necesidad de promover el respeto y la defensa de los derechos humanos, lo que incluye retos importantes como el combate de toda forma de discriminación, opresión y dominación; la lucha por la igualdad, la justicia social y la equidad de género. Estos programas de formación y prevención deben adaptarse fielmente a la realidad a la que pretenden serle útiles, por lo que, en relación con la violencia de género, se requiere un conocimiento preciso del problema en El Salvador.

Por todo lo anteriormente expuesto, la coyuntura actual en El Salvador favorece que se diseñen, inicien e implanten todo tipo de estrategias de sensibilización y prevención que vayan encaminadas a incidir directamente sobre las causas de la violencia de género. En ese sentido, la universidad, por su compromiso con la sociedad como institución de educación superior, tiene la responsabilidad de hacer propuestas. Es por ello que en esta ponencia se presentó una propuesta de investigación-acción a través del diseño de un programa de prevención de violencia de género que estuviera fundamentado en las investigaciones recientes sobre sexismo y poder (Glick y Fiske, 2001; Jackman, 1994; Pratto y Walker, 2004). Por tanto, el presente estudio pretende dar un fuerte impulso a estas estrategias preventivas en el ámbito educativo, contribuyendo a la formación y especialización de futuros profesionales de psicología para enfrentar el abordaje 
de la realidad con una nueva perspectiva, la de género. Se plantea un programa de formación en género y prevención de violencia de género que tenga una base científica en tanto en cuanto esté basado en las investigaciones recientes en la temática, y que, a su vez, se evalúe su impacto con el fin de valorar su eficacia en materia de prevención. No obstante, todavía es infrecuente encontrar una relación entre la producción científica en este campo de estudio y el desarrollo y aplicación de programas, con algunas notables excepciones (Becker y Swim, 2011; Kilmartin et al., 2008; Shields, Zawadzki y Johnson, 2011). Con este programa se pretende que ciencia y aplicación de programas vaya de la mano, demostrando la utilidad de la labor científica y académica en la resolución de problemas sociales.

Por otra parte, dentro de las estrategias de prevención de violencia de género, Coker (2004) diferencia entre prevención primaria, secundaria y terciaria. La prevención primaria es la que pretende impactar en la población general y va dirigida al diseño de estrategias que intentan que la violencia no llegue a ocurrir. Se pueden considerar ejemplos de prevención primaria las campañas en los medios de comunicación, los programas transversales en los distintos niveles educativos, los esfuerzos en políticas públicas por cambiar la legislación, las normas y estructuras sociales que incitan la violencia, etc. La prevención secundaria es la que tiene como objetivo la detección primaria de casos de violencia y su intervención, para que no siga ocurriendo esta violencia y no se convierta en un problema mayor. Un ejemplo de este tipo de prevención son los protocolos de salud para que médicos y enfermeros/as reconozcan los síntomas que pueden presentar mujeres que están sufriendo violencia, o en los mismos colegios, la formación que pueden recibir los docentes para detectar parejas que empiezan a establecer dinámicas de maltrato en sus relaciones de noviazgo. Por último, la prevención terciaria trataría de prevenir la muerte o el malestar de salud física y psicológica de una mujer que ya ha sufrido la violencia. En estos casos los esfuerzos se centrarían en atender a las víctimas, intentando, a su vez, prevenir que sigan siendo o vuelvan a ser víctimas de violencia. Las estrategias de prevención terciaria son las que se brindan en los servicios de atención psicológica de las ONG, departamentos, o instituciones que ofrecen este servicio o tienen esta función, como podría ser el Isdemu o la Procuraduría General de la República. También se consideran parte de este grupo los servicios de las casas de acogida e incluso algunos programas de intervención con maltratadores (Megías y Montañez, 2010).

El trabajo que aquí se presenta estaría dentro de la categoría de las estrategias de prevención primaria por tratarse de un programa de formación impartido en la
Universidad Tecnológica de El Salvador con alumnos y alumnas de psicología. Su fin, por tanto, fue plantear un programa de formación con perspectiva de género para futuros profesionales; no solo con el objetivo de intervenir en los mismos participantes para que no lleguen a tener relaciones violentas a futuro y sepan distinguir cuando están experimentando maltrato o discriminación por razón de género, sino también para dotarlos de herramientas y conocimientos que les permita desempeñar sus trabajos en su desempeño profesional, ya sea en servicios de atención a víctimas como en educación, salud, psicología jurídica y servicios sociales.

Finalmente, el programa de prevención que se describe a continuación se desarrolla en el marco de otras políticas e investigaciones que contribuyen a una mejor identificación y descripción de la problemática en Centroamérica, como el "Estudio de Población de Violencia de Género en El Salvador" que se está desarrollando actualmente en la cátedra de Género de la Facultad de Ciencias Sociales de la Universidad Tecnológica de El Salvador, según la metodología de la Organización Mundial de la Salud (OMS). Dicho estudio responde a un primer paso de "definición del problema" que establece la Centers for Disease Control and Prevention (CDC) (2012), empleado por el programa de "Fortalecimiento de la capacidad para la prevención primaria de la violencia contra las mujeres" en el que actualmente están trabajando la Organización Panamericana de la Salud y la Open Society Foundations, y el cual tiene como meta principal prevenir la violencia contra las mujeres y la niñez en la región centroamericana en general, y en Costa Rica, Guatemala y El Salvador en particular.

\section{Programa de formación en prevención de violencia de género}

Como ya se mencionó anteriormente, el programa que se diseñó para este estudio se incluye en el tipo de iniciativas de prevención primaria. La prevención primaria, como señalan Megías y Montañés (2010), va dirigida a la población general y puede hacerse a través de los medios de comunicación, las políticas públicas, la coeducación y los programas en centros educativos. En concreto, nuestro programa fue desarrollado en el marco de las políticas formuladas para incluir la formación sobre prevención de violencia de género en el ámbito universitario (Crees, 2008) y orientado a futuros profesionales de las ciencias sociales. Los objetivos de la intervención - como ya han sido señalados en otro trabajo de este mismo grupo de investigación (Navarro, De Lemus, Megías, Velásquez y Ryan, 2011) — fueron los siguientes: a) incrementar la conciencia sobre situaciones de desigualdad de género experimentadas en la vida diaria; b) la reducción 
de ideologías sexistas (sexismo ambivalente, estereotipos de género, masculinidad tradicional y homofobia); $\mathrm{c}$ ) proporcionar a los participantes herramientas útiles para reducir el sexismo, la violencia sexual y actitudes homófobas en intervenciones sociales. El curso de formación fue dirigido a estudiantes de Psicología posgraduados que estaban cursando su año de especialidad, y fue incluido en el programa oficial de estudios universitarios de la Universidad Tecnológica de El Salvador. Tuvo una duración de 21 horas y se impartió en seis sesiones de tres horas cada una.

Las bases en las que se sustentó el diseño del programa de prevención, en cuanto a contenidos y distribución de las sesiones, fueron las que establece el Modelo de Poder y Género de Pratto y Walker (2004), el cual se fundamenta en la idea de que la inequidad de género es un factor característico de la dominancia de los grupos en las sociedades (Pratto, 1996; Sidanius y Pratto, 1999). El modelo de Pratto y Walker propone cuatro bases de poder (obligaciones sociales, ideología, recursos y fuerza) sobre las que se fundamenta la desigualdad de género. Mientras que la ideología sexista, el control de los recursos y la fuerza proporcionan más poder a los hombres sobre las mujeres, las obligaciones sociales reducen el poder de las mujeres respecto a los hombres. Estas autoras mantienen la idea de que las cuatro bases mantienen una relación dinámica entre ellas; por tanto, para que los esfuerzos en reducir la desigualdad de poder por razón de género sean exitosos, es necesario ejercer el cambio en las cuatro bases (Navarro et al., 2011). El programa de formación incluyó los siguientes contenidos:

Sesión 1. Género y construcción social. Para vencer posibles resistencias iniciales de los participantes, esta sesión introductoria presentó evidencias de la prevalencia de la discriminación de género y la violencia hacia las mujeres en distintas partes del mundo. A su vez, en esta sesión se presentó el modelo de poder basado en el género de Pratto y Walker (2004), con el fin de enfatizar la necesidad del enfoque psico-sociológico en los esfuerzos para reducir la violencia de género.

Sesión 2. Recursos y obligaciones sociales. Numerosas evidencias fueron proporcionadas sobre el control masculino de los recursos, haciendo uso de estadísticas en varios países y enfatizando datos provenientes de El Salvador, así como las negativas implicaciones de las obligaciones sociales para las mujeres.

Sesión 3. Ideología (I). En esta sesión se presentó a los participantes la teoría del sexismo ambivalente (Glick y Fiske, 1996; 2001) y se introdujeron investigaciones sobre estereotipos de género, así como sus consecuencias para la desigualdad de género. Según Glick y Fiske (1996), el sexismo ambivalente está conformado por dos componentes claramente diferenciados pero relacionados entre sí: el sexismo hostil, que se corresponde con el sexismo tradicional, entendido como una actitud negativa hacia las mujeres, y el sexismo benévolo, que se caracteriza por una serie de actitudes positivas hacia el género femenino en el sentido de afecto positivo, protección o búsqueda de intimidad, pero que no dejan de ser sexistas por estereotipar a las mujeres y limitarlas a ciertos papeles.

Sesión 4. Ideología (II): nuevas masculinidades y actitudes hacia la homofobia. Esta sesión introdujo los nuevos enfoques sobre la deconstrucción de la masculinidad tradicional (Pescador, 2010; Vescio, 2011), descubriendo nuevas formas de "ser hombre" a través de la empatía y la expresión de emociones. En este contexto, se trabajó el problema de las actitudes homófobas como un factor vinculante con la masculinidad tradicional, así como con comportamientos en hombres relacionados con la homosexualidad. Este concepto de homofobia y prejuicio hacia la diversidad sexual se introdujo desde un enfoque interseccional hacia el género y la identidad sexual.

Sesión 5. Fuerza. En esta sesión se definió la violencia de género y se discutió con datos a escala global y otros que contextualizan el problema en El Salvador. Se analizaron las consecuencias para la salud de la violencia contra las mujeres y el tratamiento que se realiza de este problema en el ámbito social y en los medios de comunicación. A su vez, se proporcionó información sobre las leyes nacionales para sancionar la violencia de género y de las políticas llevadas a cabo para prevenirla.

Sesión 6. Diseño y aprendizaje de modelos de intervención. Esta sesión se centró en el conocimiento y aproximación a distintas herramientas de trabajo: por un lado, la intervención coeducativa en prevención primaria del maltrato a la mujer por su pareja y la violencia sexual (Ryan y Lemus, 2010), y por otro, la atención psicológica grupal de mujeres que han sufrido maltrato (Dutton, 1992; Matud, Gutiérrez y Padilla, 2005).

Como procedimiento general de implantación del programa, se siguió una metodología participativa en la cual las explicaciones teóricas fueron combinadas con el trabajo en pequeños grupos y debates. Se les pidió a los participantes que registraran experiencias personales de discriminación y fueron animados a prestar atención a episodios sexistas en sus vidas cotidianas (mediante el uso de hojas de autoregistro), que más tarde serían expuestas 
y discutidas en el grupo. Se eligió este metodología porque hay evidencias previas que indican que la atención a episodios diarios de sexismo resulta ser una estrategia eficiente para reducir creencias sexistas (Becker y Swim, 2011). La equidad de género fue promovida como una meta común para hombres y mujeres, legalmente apoyada por las instituciones, en línea con el trabajo de Allport (1954) sobre las condiciones óptimas para el contacto entre grupos. La interseccionalidad del género y la identidad nacional fueron igualmente tenidas en cuenta, considerando la idiosincrasia del país y la evolución histórica del tratamiento del género en El Salvador. Todos los conceptos trabajados y datos ofrecidos fueron contextualizados y los participantes motivados a proporcionar ejemplos específicos y temas de discusión relacionados con su experiencia personal e identidad de género en intersección con sus otras identidades sociales.

Para medir el impacto del programa, se siguió un diseño cuasi-experimental en el que el programa (i.e., participación en el taller) fue aplicado en un grupo de preespecialidad en Psicología en la Universidad Tecnológica de El Salvador; y otro grupo que recibió una formación distinta fue el de control del diseño. La muestra total estuvo compuesta, por tanto, por 67 estudiantes de esta preespecialidad. El grupo que recibió el programa estuvo compuesto por 14 hombres y 26 mujeres de edades comprendidas entre los 23 y los 66 años, mientras que el grupo control no aleatorio estuvo compuesto por un grupo de estudiantes de la misma preespecialidad que recibió otro módulo distinto al programa, formado por 9 hombres y 17 mujeres con edades comprendidas entre los 23 y los 46 años. El programa se evaluó inmediatamente después de su finalización y a los 6 meses de haberlo recibido con el fin de comprobar si los contenidos aprendidos y los cambios de actitudes pretendidos por este se mantenían con el tiempo.

Se utilizaron distintas pruebas para evaluar el cambio de actitudes y percepción de la violencia de género, y se hizo una evaluación anterior a la aplicación del programa y una evaluación posterior. Finalmente - como ya se ha mencionado, se hizo otra evaluación a los 6 meses de su aplicación. Estas pruebas fueron las siguientes:

Escenarios de malos tratos adaptados a partir de Megías, Romero-Sánchez, Durán, Moya y Bohner (2011), que describían una situación en una relación de pareja, la cual acababa en un episodio de violencia física por parte del hombre hacia la mujer en la primera de las viñetas, en un episodio de violencia psicológica en la segunda y violencia sexual en la tercera.
Inventario de sexismo ambivalente (ASI; Glick \& Fiske, 1996; en su versión española de Expósito, Moya, y Glick, 1998). Esta escala consta de 22 ítems incluidos en dos subescalas: una de sexismo hostil y otra de sexismo benévolo.

Los resultados mostraron que se produjo una influencia del programa sobre la percepción de la violencia. En relación con la culpa atribuida a la víctima, el programa tuvo un efecto sobre las mujeres participantes en este, las cuales atribuyeron menos culpabilidad a la víctima del episodio después de haber recibido el programa; y este efecto se mantuvo 6 meses después. Sin embargo, no tuvo este impacto en los hombres participantes en el programa ni en el grupo control. Por otra parte, estos resultados tan positivos para el escenario de violencia física no se dieron para el caso de violencia psicológica, en el sentido que no fue la aplicación del programa la que produjo un efecto en los participantes. Y para la violencia sexual, sí se produjo un efecto después de la aplicación del programa, es decir, los y las participantes redujeron la culpabilidad que atribuían a la víctima en el episodio de violencia sexual después de la formación, pero este efecto se debilitó a los 6 meses.

En relación con la responsabilidad atribuida al agresor por el hecho violento, hubo un efecto significativo en el caso de la violencia física entre la primera evaluación y la segunda para las mujeres, pero no para los hombres, siendo de nuevo las mujeres del grupo que recibieron el programa de prevención las que incrementaron su percepción de responsabilidad atribuida al agresor. Esto no ocurrió en el grupo control, lo que significa que el programa tuvo un efecto en la conciencia y la percepción de los participantes. Sin embargo, no hubo efectos para la evaluación a los 6 meses, lo que quiere decir que, una vez trascurrido el tiempo, los participantes volvieron a utilizar justificaciones para la violencia que exculpan al agresor, probablemente influidos por los mitos que existen en torno a la violencia de género y que en el ámbito cultural la justifican. No obstante, como en el caso anterior, los resultados no fueron tan satisfactorios para la violencia psicológica y sexual.

La influencia del programa sobre las medidas ideológicas también fue significativa. Como ya se mencionó, se evaluó el sexismo ambivalente antes y después del programa, buscando un cambio de actitudes tanto en el sexismo hostil como en el benévolo. En términos generales, el programa tuvo un impacto en el cambio de actitudes de los y las participantes, pero estos resultados fueron mucho más positivos en relación con el sexismo hostil; y el cambio de actitudes sexistas hostiles se mantuvo a los 6 meses de haber recibido el programa. En este caso no hubo diferencias significativas entre hombres y mujeres y no se produjeron 
dichos cambios en el grupo control, lo que significa que los cambios se debieron efectivamente a la aplicación del programa. Por otra parte, respecto al sexismo benévolo, mostró un patrón similar de reducción de las actitudes sexistas benévolas tras la participación en el programa, efecto que no se mantuvo a los 6 meses.

\section{Conclusiones}

El programa de formación y prevención de violencia de género que se presentó en esta ponencia tuvo buenos resultados, en tanto en cuanto se obtuvo cambios de actitudes en los participantes, actitudes que han sido identificadas como parte de las causas de la violencia de género. No en vano $-\mathrm{y}$ como ya se señalaba en la Declaración de Beijing (ONU, 1995) - el poder tiene un papel fundamental en la explicación de las causas de la violencia de género; y la desigualdad de poder por razón de género, su análisis y discusión, es la base del programa que se diseñó. Siguiendo el Modelo de Poder de Pratto y Walker (2004), se articularon los contenidos del programa y se midieron los resultados en cada uno de los componentes. Algunos resultados que se obtuvieron fueron muy positivos, ya que la participación en este programa permitió aumentar la percepción de violencia de género entre los participantes a través de la culpa que se atribuye a la víctima y la responsabilidad que se deposita sobre el agresor, que al fin y al cabo son las bases de la justificación social de este tipo de violencia. Por otra parte, se consiguió cambiar actitudes reduciendo sus actitudes sexistas hostiles y manteniendo estos efectos 6 meses después de haber participado en el programa. En consonancia con los resultados encontrados por Navarro et al. (2011) en los que se pudo constatar un cambio en las actitudes sexistas hostiles y benévolas, el papel masculino tradicional y las actitudes homófobas justo después de haber participado en el programa, se prueba su efectividad para el cambio de actitudes, la formación en género y la prevención de la violencia de género aún cuando han pasado 6 meses de haberlo recibido, lo que demuestra que el programa constituye una herramienta valiosa no solo de formación, sino que también de prevención en los mismos participantes.

Finalmente, es importante resaltar que este modelo de programa que ha sido probado científicamente y medido sus resultados positivos, podría ser el inicio de un plan de prevención de violencia de género más amplio que adoptaran las políticas públicas haciendo cumplir de esta forma la Ley especial integral para una vida libre de violencia para las mujeres (LEIV) que fue aprobada el 25 de noviembre de 2010, y que tiene el firme propósito de prevenir, pero que sin embargo aún resulta deficiente en recursos, formación y diseño de estrategias.

\section{Referencias}

Allport, G.W. (1954). The nature of prejudice. Cambridge, MA: Addison-Wesley.

Asamblea Legislativa República de El Salvador (2011). Ley especial integral para una vida libre de violencia para las mujeres (LEIV). Disponible en: www.asamblea.gob. $\mathrm{sv} / .$. legislativo/...legislativos/ley-especial-integral.

Becker, J.C. y Swim, J.K. (2011). Seeing the Unseen: Attention to Daily Encounters With Sexism as Way to Reduce Sexist Beliefs. Psychology of Women Quarterly, 35, $227-242$.

Buvinic, M.; Morrison, A. y Shifter, M. (1999). Violence in Latin America y Caribbean: a framework for action. Washington, DC: Inter-American Development Bank.

Centers for Disease Control and Prevention (CDC) (2012). Extraído de: ttp://www.cdc.gov/ncipc/ dvpPublichealthApproachTo

Coker, A.L. (2004). Primary prevention of Intimate Partner Violence for women's health. A response to Plichta. Journal of nterpersonal Violence, 19, 1324-1334.

Crees (2008). "Declaración de la conferencia regional de la Educación Superior en América Latina y el Caribe". Extraído de: http://www.iesalc.unesco.org.ve/docs/ wrt/declaracioncres espanol.pdf

Dutton, M.A. (1992). Empowering and healing the battered woman: A model for assessment and intervention. New York: Springer.

Expósito, F.; Moya, M. y Glick P. (1998). "Sexismo ambivalente: Medición y correlatos". ["Ambivalent sexism: measurement and correlates"]. Revista de Psicología Social, 13(2), 159-169.

Fesal (2008). Encuesta Nacional de Salud Familiar. Informe Final. Extraído de: http://www.fesal.org.sv/

Garaizabal, C. y Vázquez, N. (1994). El dolor invisible. Una experiencia de grupos de autoayuda con mujeres salvadoreñas. Madrid: Talasa.

Glick, P. y Fiske, S.T. (1996). "The Ambivalent Sexism Inventory: Differentiating hostile and benevolent sexism". Journal of Personality and Social Psychology, 70, 491-512.

Glick, P. y Fiske, S.T. (2001). "Ambivalent sexism". In M. P. Zanna (Ed.), Advances in experimental social psychology (pp. 115-188). San Diego: Academic Press.

Hume, M. (2008). "The myths of violence: gender, conflict and community in El Salvador". Latin American Perspectives, 35, 59-76. 
Isdemu (2011). "Política Nacional de las Mujeres". San Salvador, Isdemu, 2011. Extraído de:http://www. isdemu.gob.sv/index.php?option=com phoca download \&view=category \&id=46\%3Apoliticaspublicas\&ltemid=234\&lang=es

Jackman, M.R. (1994). The velvet glove. Berkeley: University of California Press.

Kilmartin, C.T.; Smith, T.; Green, A.; Heinzen, H.; Kuchler, M. y Kolar, D. (2008). "A real-time social norms intervention to reduce college mens' sexism". Sex Roles, 59, pp. 264-273.

Matud, M.P.; Padilla, V. y Gutiérrez, A.B. (2005). Mujeres maltratadas por su pareja. Guía de tratamiento psicológico. Madrid: Minerva Ediciones.

Megías, J. y Montañéz, P. (2010). “Prevención primaria de la violencia de género". En E. Ryan y S. de Lemus (Eds.) Coeducación. Propuestas para alcanzar la igualdad de género desde las aulas (pp. 127-163). Granada: Editorial Universitaria.

Megías, J.L.; Romero, M.; Durán, M.; Moya, M. y Bohner, G. (2011). "Spanish validation of the acceptance of modern myths about sexual aggression scale (Ammsa)". The Spanish Journal of Psychology, 14, 912-925.

Navarro, L.; de Lemus, S.; Megías, J.L.; Velásquez, M.J. y Ryan, E. (2011). "Programa universitario de formación en prevención de violencia de género en El Salvador". En: M. Jiménez Ramírez, V. Robles Sanjuán, F. Añaños Bedriñana y F.J. del Pozo Serrano (Coords.), Educación para la igualdad: reflexiones y Propuestas (pp. 211221). Granada: Editorial Natívola.

Organización de Naciones Unidas (1995). Plataforma de Acción de Beijing y la violencia contra las mujeres. Recuperado el 16 de noviembre de 2006 de: http:// www.choike.org/nuevo/informes/3826.html.

Organización Mundial de la Salud (2005). “Estudio multipaís de la OMS sobre salud de la mujer y violencia doméstica contra la mujer: primeros resultados sobre prevalencia, eventos relativos a la salud y respuestas de las mujeres a dicha violencia: resumen del informe". Recuperado el 10 de noviembre de 2006 de: http:// www.who.int/gender/violence/who_multicountry_ study/summary_report/summaryreportSpanishlow. pdf

Ormusa (2012). Observatorio de la violencia de género contra las mujeres. Extraído de: http:// observatoriodeviolencia.ormusa.org/

Pan American Health (2012). Violence Against Women. In latin american and the caribbean: a comparative analysis of population-based data from 12 countries.

Pescador, E. (2010). "Reconstrucción de las masculinidades". En: E. Ryan y S. de Lemus (Eds.) Coeducación. Propuestas para alcanzar la igualdad de género desde las aulas (pp. 101-126) Granada: Editorial Universitaria. Pratto, F. (1996). "Sexual politics: The gender gap in the bedroom, the cupboard, and the cabinet". In: D. M. Buss y N. Malamuth (Eds.), Sex, power, and conflict: Evolutionary and feminist perspectives (pp. 179-230). NY: Oxford University Press.

Pratto, F. y Walker, A. (2004). "The bases of gendered power". In: A. H. Eagly, A. E. Beall y R. J. Sternberg (Eds.), The Psychology of Gender (2nd ed) (pp. 242-268). New York: The Guilford Press.

Red Feminista Frente a la Violencia Contra las Mujeres -RED FEM- (2012). Ley Especial para una vida Libre de Violencia para las mujeres. Con comentarios. San Salvador: Impresos Continental, S.A.

Ryan, E. y de Lemus, S. (2010). Coeducación. Propuestas para alcanzar la igualdad de género desde las aulas. Granada: Editorial Universidad de Ganada.

Shields, S.A.; Zawadzki, M.J. y Johnson, R.N. (2011). "The impact of a workshop activity for gender equity simulation in the academy (WAGES-Academic) in demonstrating cumulative effects of gender bias". Journal of Diversity in Higher Education, 4, 120-129.

Sidanius, J. y Pratto, F. (1999). Social dominance. An intergroup theory of social hierarchy and oppression. New York: Cambridge University Press.

Vescio, T.K.; Schlenker, K.A. y Lenes, J.G. (2010). "Power and Sexism". En: A. Guinote y T.K. Vescio. The Social Psychology of power (pp.363-380). London: Guilford Press. 